\title{
La alopecia androgénica femenina: ¿cuáles son las principales preguntas de mis pacientes?
}

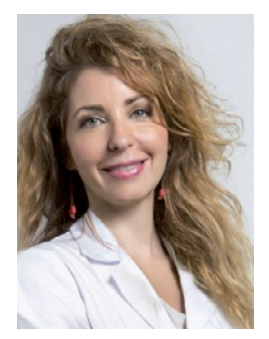

Elena González-Guerra Profesora asociada de Dermatología Médico-Quirúrgica y Venereología. Departamento de Medicina. Universidad Complutense de Madrid. Médico adjunto.

Servicio de Dermatología. Hospital Universitario Clínico San Carlos. Madrid. Directora del Máster en Dermofarmacia y Formulación Cosmética. Universidad Internacional de La Rioja.

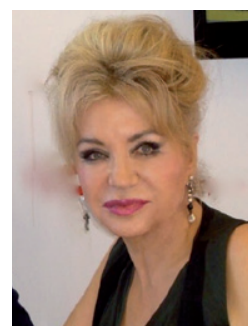

\section{Aurora Guerra-Tapia}

Profesora titular de Dermatología. Departamento de Medicina. Facultad de Medicina. Universidad Complutense de Madrid.

Exjefa de Sección

de Dermatología.

Hospital Universitario 12 de Octubre. Madrid. Directora del Máster en Dermofarmacia y Formulación Cosmética. Universidad Internacional de La Rioja.
La alopecia es un problema dermatológico y estético que afecta psicológicamente a quien lo padece. El cabello conforma en gran medida la autoimagen, base, a su vez, de la autoestima, por lo que su disminución o ausencia revierte inevitablemente en el bienestar emocional ${ }^{1}$. Diferentes estudios han demostrado que la mujer sufre en mayor medida dicha repercusión, incluso con grados leves del proceso, debido a la evidente presión social de la necesidad de una estética femenina favorable frente a la masculina ${ }^{2,3}$.

Cuando una paciente acude a nuestra consulta con una alopecia androgénica femenina, casi siempre muestra un alto grado de ansiedad tanto por el diagnóstico y la evolución de su problema, como por las informaciones obtenidas de diferentes medios sociales, que, a menudo, erróneas o exageradas, solo sirven para aumentar su angustia. Es fundamental, por ello, ocupar todo el tiempo necesario en informar suficientemente, y responder a las preguntas que habitualmente nos hacen ${ }^{4}$.

Así, comenzamos por informar de que este tipo de alopecia que hemos diagnosticado consiste en una disminución lenta y progresiva de la densidad y grosor de su cabello, que se convierte en vello. Los ciclos foliculares de crecimiento y reposo (anágeno y telógeno) producen cada vez pelos más cortos y de menor diámetro, que, en estadios finales, son casi invisibles. Pero no hay cicatrización, ni pérdida definitiva de folículos, salvo en casos muy avanzados. Es, por lo tanto, una alopecia reversible.

-Yo creía que las mujeres no nos quedábamos calvas. Y no veo otras como yo. A veces creo que solo me pasa a mí. ¿Es una enfermedad rara?

La alopecia androgénica femenina es muy frecuente, y afecta a más del $40 \%$ de las féminas a lo largo de su vida, aumentando con la edad. Lo que ocurre es que la forma de pérdida de pelo es diferente a la del varón 
(fig. 1), y las herramientas cosméticas de las que después hablaremos disimulan su presencia.

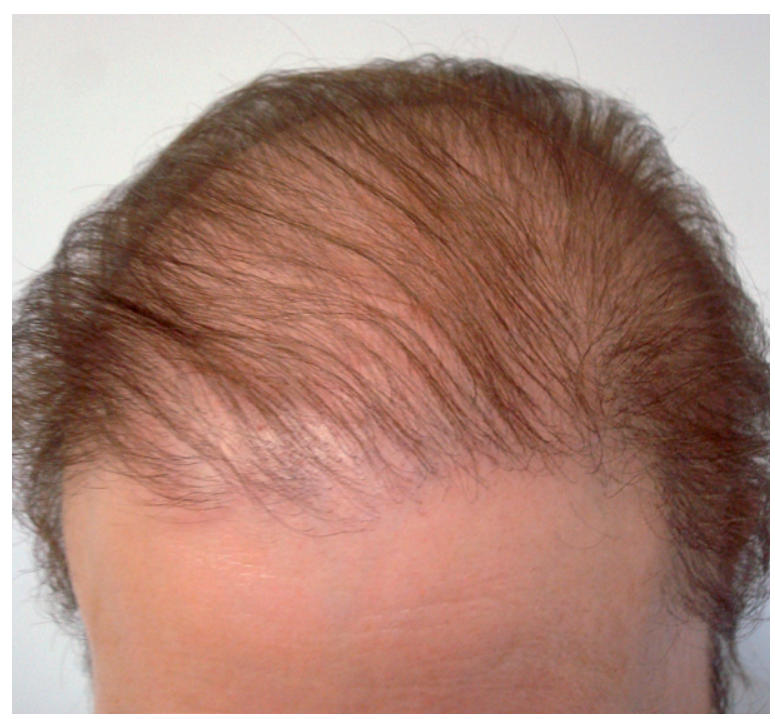

Figura 1. La miniaturización del cabello en la mujer suele afectar de forma difusa a todo el cuero cabelludo, como en este caso.

—Pero ¿por qué no me hace más pruebas? He visto que en algunos sitios hacen un análisis del pelo y del cuero cabelludo.

La exploración de la alopecia androgénica se realiza mediante la inspección meticulosa, que habla de las características macroscópicas del pelo, tales como el color, el brillo, la densidad, la longitud y la forma, así como el estado del cuero cabelludo; con la palpación, podemos conocer la textura (áspero, suave, graso, seco, grueso, fino) y la fragilidad; gracias al pellizcamiento o signo de Jacquet, sabemos de una forma aproximada si el cuero cabelludo contiene muchos o pocos folículos activos; si traccionamos desde la base hacia el extremo terminal de un mechón (signo de Sabouraud), conoceremos si hay una caída excesiva según el número de cabellos desprendidos, lo que hablará de un posible efluvio telógeno añadido. La tricodermatoscopia (observación del cuero cabelludo con un dermatoscopio) ampliará la información sobre el estado de los folículos pilosos y su posible recuperación. Otras pruebas como el tri- cograma - consistente en la observación al microscopio de los extremos de un mechón de 50 a 100 pelos arrancado con una pinza o portagujas-, el fototricograma - que compara una zona de cuero cabelludo en diferentes momentos evolutivos-, la biopsia o el análisis químico del pelo —útil en intoxicaciones y anomalías genéticasno son necesarios en la práctica habitual en este tipo de alopecia ${ }^{5}$.

\section{- ¿Por qué me ha ocurrido a mí?}

En las causas de la alopecia androgénica, existe un factor genético o de herencia poligénica multifactorial, y otro hormonal, por las hormonas masculinas o andrógenos. Ambos factores determinan la intensidad y la evolución de cada caso.

\section{-Pero ¿las mujeres tenemos andrógenos?}

La mujer produce andrógenos en los ovarios, las cápsulas suprarrenales, y algunos tejidos no endocrinos, como, por ejemplo, la piel. Los ovarios segregan el $20 \%$ de la testosterona, el 20-30\% de la deshidroepiandrosterona, el $10 \%$ del sulfato de deshidroepiandrosterona y el $60 \%$ de la delta4-androstenodiona. Las glándulas suprarrenales segregan el $30 \%$ de la testosterona, el $40 \%$ de la androstenodiona, el $70 \%$ de la deshidroepiandrosterona, el $90 \%$ del sulfato de deshidroepiandrosterona y cantidades variables de andrógenos como productos intermedios. En la piel, el hígado y el músculo esquelético, la androstenodiona y el sulfato de deshidroepiandrosterona se convierten en testosterona, representando el otro $50 \%$ que complementa al producido por los ovarios y las glándulas suprarrenales. Por lo tanto, las mujeres también producen andrógenos. Para que la acción androgénica se lleve a cabo, es preciso que las hormonas se unan a receptores específicos, que se encuentran localizados en estructuras concretas como el folículo piloso y la glándula sebácea. La inducción de pelo terminal a velloso y finalmente atrófico (alopecia androgénica) se promueve por los andrógenos, en las zonas del cuero cabelludo donde existen mayor número de receptores para ellos, gracias a la acción de la $5 \alpha$-reductasa ${ }^{6}$. 
- ¿Por qué es diferente la alopecia androgénica de la mujer y la del hombre?

Los diferentes patrones clínicos en la mujer respecto al varón se deben a la diferencia en los niveles y el patrón de distribución de la enzima $5 \alpha$-reductasa (que transforma la testosterona en dihidrotestosterona), la aromatasa y los receptores de andrógenos en los folículos pilosos de la mujer (difuso) (v. fig. 1) respecto del hombre (frontal y parietal). Por ejemplo, los receptores androgénicos del área frontal femenina son solamente un $40 \%$ de los que existen en el hombre ${ }^{6}$. No obstante, algunas mujeres pueden tener un patrón similar al masculino (fig. 2).

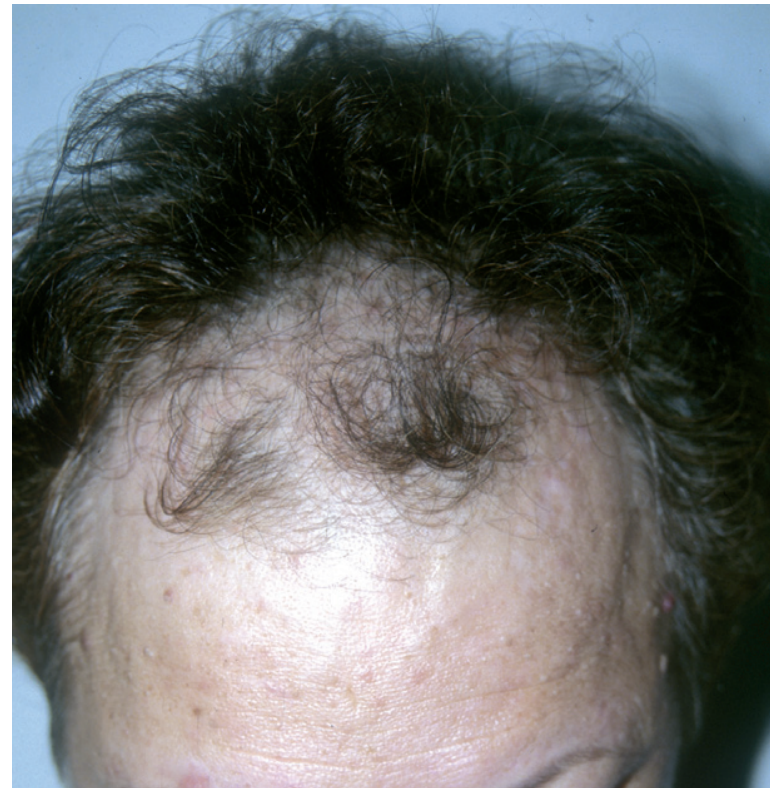

Figura 2. Patrón masculino de la alopecia androgénica femenina.

\section{—¿Y me tengo que hacer análisis?}

Es conveniente hacer un estudio complementario hormonal, de depósitos de hierro (ferritina) y algunos otros parámetros que dependen de las preguntas y exploración general que haga el dermatólogo, con la finalidad de descartar otras alteraciones como el síndrome del ovario poliquístico, que cursa con alteraciones menstruales, seborrea, hirsutismo, acné persistente... Pero a veces no es necesario. Su dermatólogo decidirá lo mejor en su caso.

\section{—¿La grasa del cuero cabelludo hace que se caiga el pelo?}

La asociación de sebo y alopecia es habitual, ya que la glándula sebácea es igualmente dependiente en su función de los andrógenos. Pero no produce alopecia, sino que la acompaña.

\section{—¿Cuál es el mejor tratamiento?}

La alopecia androgénica femenina necesita un tratamiento múltiple. Uno de ellos con indicación aprobada para este proceso es el minoxidil tópico, que tiene una acción activadora inespecífica del desarrollo de los folículos pilosos, prolongando el período de crecimiento, deteniendo la miniaturización y consiguiendo en muchos casos su recuperación ${ }^{7}$. Con el uso adecuado, mañana y noche -y, preferiblemente, al $5 \%$ - , se consigue hasta un $45 \%$ de repoblación, con un pico de crecimiento a las 16 semanas. A las 24 semanas de su aplicación, el grosor de los cabellos llega a aumentar hasta un $30 \%$. Sin embargo, no produce neogénesis folicular, por lo que no actúa donde no existen folículos.

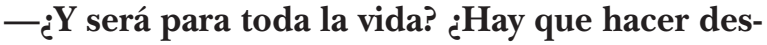
cansos?

El tratamiento con minoxidil hay que mantenerlo de forma indefinida, ya que, si se suspende, se pierde el efecto beneficioso conseguido. Si se interrumpe la aplicación, desaparece su efecto entre tres y seis meses después de la suspensión, por la transición brusca a la fase telógena de todos los cabellos que ampliaron su fase de crecimiento o anágena. Por lo tanto, no se deben hacer descansos en este tratamiento ${ }^{7}$.

\section{—¿Lo puedo usar si estoy embarazada?}

No se recomienda su uso durante el embarazo, aunque, en los estudios realizados en animales, no se ha detectado un efecto nocivo en el desarrollo embrionario/fetal, parto o durante el desarrollo posnatal, ni la experiencia en la práctica real 
ha demostrado alteraciones. Se excreta por la leche materna, por lo que tampoco se indica en la lactancia ${ }^{8}$.

\section{—¿Qué otros tratamientos puedo hacer?}

Algunas mujeres pueden tener hiperandrogenismo con otros signos clínicos como hirsutismo, acné resistente al tratamiento, galactorrea, infertilidad o alteraciones de la menstruación, aunque la mayoría no tienen datos clínicos ni de laboratorio de exceso de andrógenos. En esos casos, son útiles los antiandrógenos orales, mediante anticonceptivos específicos u otras sustancias (acetato de ciproterona, espironolactona, finasterida) que controlen el excesivo aprovechamiento de sus andrógenos. Sus pros y sus contras le serán explicados según cada caso $^{9}$.

\section{—¿Qué cuidados higiénicos y cosméticos debo} hacer?

Debe lavar el cabello siempre que esté sucio con champú que limpie sin agredir. El lavado frecuente no produce alopecia ni efecto de rebote de la grasa o seborrea. Cuando existe un trastorno del cuero cabelludo, como la caspa o dermatitis, debe ser un champú específico. El secado con secador debe utilizar temperaturas moderadas. El sobrecalentamiento del agua sobre el pelo puede producir la fractura de este, con atrapamientos de bolsas de aire en su interior, dando lugar al pelo en burbuja. Las lacas, espumas fijadoras y gominas tienen la misión de dar y mantener la forma elegida para el cabello, y no son perjudiciales.

\section{- ¿Me puedo hacer un moldeado permanente o teñirme? \\ El cambio duradero de la forma del cabello, me- diante la aplicación de sustancias químicas (tiogli- colatos) que rompen los enlaces disulfuro o la aplicación de tintes puede dañar la estructura del cabello, especialmente, si se mantiene un excesivo tiempo de exposición o si se combinan entre sí en un corto período de tiempo. Sin embargo, no pro- ducen alopecia, pues el pelo dañado se sustituirá por pelo nuevo y sano con el tiempo ${ }^{10}$.}

\section{— ¿Tengo que tomar vitaminas?}

La administración de hierro, biotina, cinc, vitamina B y otros nutricosméticos es útil en la presencia de efluvio telógeno añadido, o de carencias demostradas $^{11,12}$. También los extractos lipídicos de Serenoa repens y de Pygeum africanum son capaces de ejercer una acción inhibidora de la $5 \alpha$-reductasa de tipo 1 y de tipo 2.

\section{- ¿Me puedo hacer un trasplante capilar?}

Todo depende de su forma clínica. Si tiene una buena zona dadora - generalmente, occipital (v. fig. 2)-, el resultado será bueno. Pero, si es una alopecia difusa (v. fig. 1), no será posible hacer un microinjerto.

\section{—¿Cómo puedo disimular mi alopecia?}

Hay diversas herramientas cosméticas que pueden ayudar: las microfibras de queratina, el sistema de integración capilar ${ }^{13}$, el sistema FAS (del inglés, follicular alternative system), las prótesis (pelucas) parciales o totales... Forman parte de la estrategia para evitar la ansiedad y conseguir un buen aspecto de forma transitoria mientras el tratamiento hace su efecto o en los casos de mal pronóstico.

Podríamos alargar este tema indefinidamente. Cada mujer tiene sus inquietudes, y la comunicación seguirá siendo precisa a lo largo de las sucesivas consultas. Pero, tal vez, este resumen puede ayudar a tranquilizar a algunas de ellas.

\section{BIBLIOGRAFÍA}

1. Martínez-González MC, Martínez-González RA, Guerra-Tapia A. Esthetic dermatology and emotional well-being according to gender. J Cosmet Dermatol. 2018;17(3):410-6.

2. Zehravi M, Maqbool M, Ara I. Depression and anxiety in women with polycystic ovarian syndrome: a literature survey. Int J Adolesc Med Health. 2021. [En prensa].

3. Martínez-González C, Guerra-Tapia A, González-Guerra E. El paciente de dermatología estética: factores psicológicos. Más Dermatol. 2021;35:7-16.

4. Martínez-González MC, Martínez-González RA, Guerra-Tapia A. Key communication skills in cosmetic dermatology: a 3-pillar model. Actas Dermosifiliogr (Engl Ed). 2019;110(10):794-9.

5. González-Guerra E, López-Bran E. Protocolo diagnóstico de la alopecia. Medicine. 2018;12(48):2864-7.

6. Ho CH, Sood T, Zito PM. Androgenetic alopecia. En: Stat Pearls [Internet]. Treasure Island (FL): Stat Pearls Publishing; 2021.

7. Guerra-Tapia A, González-Guerra E. Minoxidil. Tratamiento eficaz de la alopecia. Madrid: Editorial IMC; 2016. 
8. Guerra-Tapia A, González-Guerra E. Alopecia de patrón femenino (FAGA). En: Guerra-Tapia A, González-Guerra E. Algoritmos terapéuticos en dermatología básica. Madrid: Editorial Médica Panamericana; 2013. p. 117-21.

9. Guerra-Tapia A, González-Guerra E. Finasterida tópica y alopecia androgenética masculina y femenina. Más Dermatol. 2017;27:3-5.

10. Guerra-Tapia A, González-Guerra E. Hair cosmetics: dyes. Actas Dermosifiliogr. 2014;105(9):833-9.
11. Guerra-Tapia A, González-Guerra E, Borrás Schierloh JM. Alopecia androgénica femenina. Nuevas herramientas terapéuticas frente a los factores fisiopatológicos implicados: hormonal, oxidativo e inflamatorio. Más Dermatol. 2017;27:21-33.

12. González-Guerra E, Guerra-Tapia A. Suplementos nutricionales y piel. Más Dermatol. 2021;33:31-6.

13. Guerra-Tapia A, González-Guerra E, De la Cruz-Bertolo J. Alopecia female hair integration system: a study of the psychological impact. Med Cutan Iber Lat Am. 2012;40(4):103-8. 